\section{Los públicos internos en la construcción de la imagen corporativa}

Andrea Paula Lojo ${ }^{(*)}$

\begin{abstract}
Resumen: Este artículo reflexiona acerca de los públicos de la organización como destinatarios de la gestión de las relaciones públicas, específicamente el público interno, con el objetivo de señalar su rol e importancia en las organizaciones; tanto como partícipes del proceso de origen y obtención de información, como también en la construcción de las estructuras mentales cognitivas de los públicos externos que antecede a la generación de los vínculos organizacionales. Para esto, necesariamente se vincula comunicación interna, públicos internos, cultura organizacional y comunicación corporativa. Y cómo éstos impactan en la construcción de una imagen positiva. Finalmente, profundiza sobre las nuevas tecnologías y el cambio de paradigma comunicacional, en el que el público interno definitivamente asume el rol de vocero.
\end{abstract}

Palabras claves: Comunicaciones Corporativas - Comunicación Empresaria - Comunicación Institucional - Comunicación Interna - comunicadores - Dircom - Imagen Corporativa - nuevas tecnologías - público externo - público interno - Relaciones Públicas - vocero.

[Resúmenes en inglés y portugués y currículum en la página 94]

\title{
Introducción
}

"Las relaciones públicas son una actividad triple que consiste en: información dada al público, persuasión dirigida al público para modificar sus actitudes y acciones; y un esfuerzo por integrar las actitudes y acciones de una compañía o institución con sus públicos y de los públicos con la compañía o institución" (Bernays, 1958, p. 11).

La que antecede es una definición sobre lo que significan las relaciones públicas para Edward Bernays, a quien muchos consideran el padre de esta disciplina. Sus palabras continúan vigentes hasta el día de hoy. Como se puede apreciar, pone el acento en el abordaje de los públicos de la organización como destinatarios de la acción de las relaciones públicas. Este foco se encuentra tanto en relación a la información que se brinda, como en la intencionalidad de persuadir a los públicos para que modifiquen sus acciones y un esfuerzo por integrar de manera bidireccional a los públicos y las organizaciones.

En este contexto, el objetivo del artículo es señalar el rol y la importancia específica que tienen los públicos internos de las organizaciones: tanto como partícipes del proceso de origen y obtención de información, como también en la construcción de las estructuras mentales cognitivas de los públicos externos que antecede a la generación de los vínculos con las organizaciones. 


\section{Las Relaciones Públicas y la Imagen Corporativa}

Tal como expresa Joan Costa (2009) vemos en la figura del DirCom, al profesional que comprende cuales son los conocimientos necesarios, las habilidades de dirección, la estrategia y el liderazgo que se requiere para la gestión de las comunicaciones globales y los valores intangibles de las empresas, instituciones y organizaciones.

Hoy esa figura está en alza, y son los relacionistas públicos, aquellos profesionales, formados para responder a esta demanda contemporánea del DirCom: quienes tienen como parte de su misión, la construcción de una imagen corporativa pública, notoria y valorada.

El aporte de esta imagen corporativa de las organizaciones deberá:

- Crear valor: para otorgar a la organización un sentido positivo, un ser social; que pueda ser asumido como imagen de la entidad en la mente de los públicos.

- Facilitar la diferenciación: forjar una imagen que aporte un valor diferencial, de modo que los públicos consideren a la organización como una opción/alternativa respecto a otras organizaciones.

Paul Capriotti (2009) agrega otros beneficios importantes que aporta la imagen corporativa, entre los que se detacan:

-Vender mejor: si la empresa posee una imagen positiva que supera a la de sus competidores, podrá vender sus productos y/o ofrecer sus servicios a un precio mayor, dado que sus consumidores/ clientes están dispuestos a pagar un 'plus de marca'.

- Atraer mejores trabajadores: aquella organización que posea la mejor imagen corporativa de su sector se convertirá en referente como empleador y será considerada como un buen lugar donde trabajar. De este modo, podrá atraer con mayor facilidad a los mejores trabajadores y retener a sus talentos internos.

En estos cuatro beneficios de una adecuada y sólida imagen corporativa se puede incluir el comportamiento y el nivel de conversaciones que se establecen entre los miembros de una organización: primero en el entorno interno para luego proyectarse hacia el entorno externo. Veamos en los siguientes capítulos cómo los Públicos Internos se relacionan con el proceso de formación de la Imagen Corporativa.

\section{La Imagen Corporativa y la Comunicación de la Organización}

La Imagen Corporativa se forma en la mente de los públicos a partir del procesamiento de la información que recibe sobre la organización. Por eso, Imagen se relaciona con Recepción.

La recepción forma parte del proceso de comunicación de una organización, que incluye todo lo que la misma dice a sus públicos, ya sea por las acciones de comunicación propiamente dicha como por su conducta corporativa. 


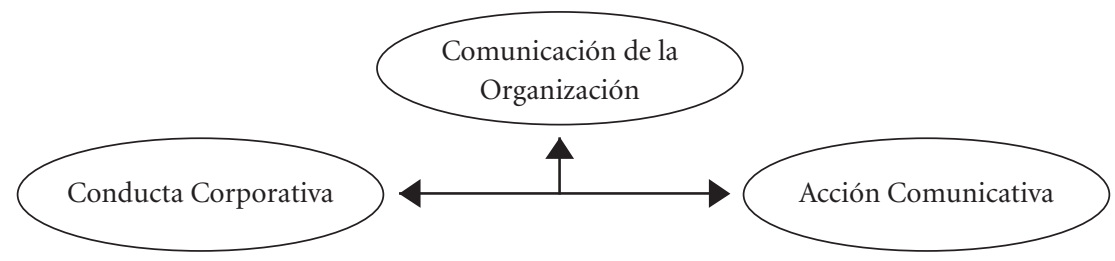

Figura 2: La Comunicación en la Organización.

Fuente: Capriotti, P. (1999). Planificación estratégica de la imagen corporativa. Barcelona: Ariel.

Pero el proceso de formación de esta imagen es un proceso complejo que tiene diferentes etapas. En su vinculación con el público interno es oportuno mencionar la etapa de origen de la información para la construcción de esta imagen positiva y también cómo los públicos obtienen esta información.

\section{Origen de la Información}

Para los públicos de la organización, todos los mensajes que recibe sobre la misma contribuyen a la formación de su imagen. Es por eso que las organizaciones se preocupan cada vez más por reconocer y coordinar las diferentes fases de su comunicación hacia el entorno.

En línea con Capriotti al afirmar que estas fases de comunicación se componen tanto por el "hacer saber" como por el "saber hacer".

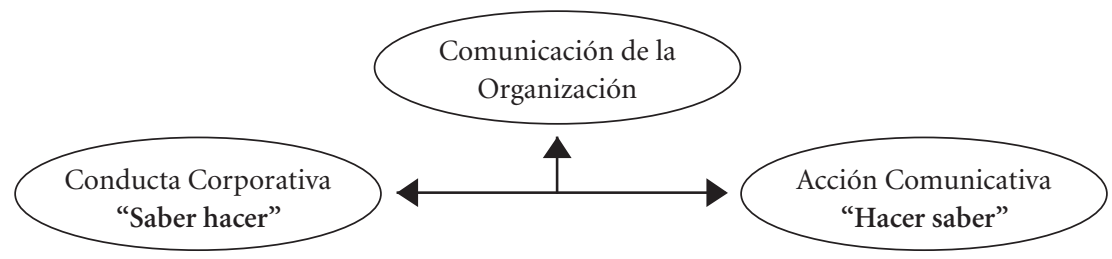

Figura 3: La Comunicación en la Organización.

Fuente: Capriotti, P. (1999). Planificación estratégica de la imagen corporativa. Barcelona: Ariel.

Este "hacer saber" o acción comunicativa incluye todas las actividades de comunicación, voluntarias y formalmente planificadas de la organización, que tienen como objetivo principal transmitir mensajes hacia los diferentes públicos, brindando información sobre la organización.

Se realiza una tipificación de estas comunicaciones, se puede mencionar la comunicación institucional, la comunicación de marketing, la comunicación de marca, a la comunicación interna, entre otras.

Y al abordar el "saber hacer" o conducta corporativa estamos ante la presencia de la actividad propia de la organización: aquello que hace o no hace. Y estas acciones u omisiones también forman parte de la construcción de la imagen corporativa y además, de la evaluación que los públicos 
hacen de la organización en cuanto se vinculan con sus intereses respecto a lo que "debería hacer o no hacer" la misma.

Entonces, si tomamos las palabras de Joan Costa podemos decir que la acción es también comunicación. Y a través de las acciones, la organización expresa claramente sus valores, o sea cuáles son sus principios profesionales y de relación que representan cómo lleva adelante sus negocios. Capriotti (1999) diferencia tres tipos de comportamientos en las organizaciones: la conducta comercial, la conducta institucional y la conducta interna.

Vemos entonces, en esta fase de origen de la información para la construcción de la imagen corporativa, cómo y dónde aparecen los Públicos Internos de las organizaciones.

Por un lado, se encuentra la Comunicación Interna, que se dirige a los empleados de la organización; o sea, a sus Públicos Internos. Este tipo de comunicación corporativa tiene como objetivo principal dirigir la Cultura Organizacional hacia la dirección deseada; o sea, hacia la concreción de los objetivos que la organización se propone. Una eficaz gestión de la Comunicación Interna debe alinear e involucrar a los empleados con estos objetivos, armonizar el clima de la organización para generar el ambiente adecuado de trabajo, promover el cambio o refuerzo de comportamientos que permita lograr los fines y finalmente, mejorar la productividad. Por otro lado, se encuentra la Conducta Interna, que representa la forma en que la organización se comporta respecto a sus miembros. Y este comportamiento se produce en diferentes niveles. En primer lugar, se puede ubicar el comportamiento del top management (alta gerencia) de la organización, quienes no sólo comunican a través de lo que dicen sino sobre todo, a través de lo que hacen, de sus actos. Y estos actos encuentran una formalización en la implementación de las políticas, las normas, los procedimientos; los cuales determinan, por ejemplo, cómo la organización promociona a sus empleados, cómo los selecciona, los capacita, evalúa su desempeño, recompensa las contribuciones y sanciona los incumplimientos...Todas ellas, acciones tendientes a establecer una adecuada satisfacción laboral que genere una evaluación positiva. Otro nivel corresponde a las pautas que permiten el correcto funcionamiento de la organización, aquellas formas de hacer que le permiten lograr sus objetivos y metas. Se trata entonces de la estructura formal que adopta la organización y en la cual los empleados desarrollan sus tareas. En este sentido, la estructura representa a la organización, es su marco organizativo y dice mucho de sí misma en el ámbito interno. Se trata de cómo se hacen las cosas en la organización. Y finalmente, nos encontramos con lo que se manifiesta a través de la filosofía y la cultura corporativa: entendiendo estos conceptos como la misión, visión y valores de la organización y la forma cómo se comportan sus miembros. Esta definición de sí misma en cuanto a por qué existe, para qué es necesaria, a qué se dedica, cuál es su rumbo, que "debe ser" y "cómo" llegará a serlo, es un elemento vital para la formación de la imagen corporativa; y son los miembros de la organización, los primeros que deben asumir un comportamiento acorde a esta filosofía corporativa para lograr la coherencia organizacional entre el hacer y el decir.

De este modo, Comunicación Interna y Conducta Interna conforman la Comunicación Corporativa Organizacional que se vincula con los Públicos Internos.

La visión tradicional de la gestión de la Comunicación y la Conducta Interna se centra en cómo planificar ambas instancias para lograr la construcción de una imagen positiva de la organización en sus Públicos Internos; en el mismo nivel que se gestiona la construcción de una imagen positiva en sus Públicos Externos.

Una visión actual de la gestión de la Comunicación y de la Conducta Interna debiera tener 
en cuenta a los Públicos Internos como partícipes en el proceso de formación de la Imagen Corporativa dado que los miembros de la organización se encuentran en un lugar especial de influencia hacia los Públicos Externos debido al nivel de información y conocimiento que poseen de la organización misma.

Desde este enfoque, el objetivo es que las organizaciones establezcan una adecuada Conducta Interna y planifiquen una eficaz gestión de la Comunicación Interna, para promover el desarrollo de un entorno favorable y satisfactorio de trabajo. De modo que los empleados asuman sin contradicciones la Misión, la Visión y los Valores de la Organización y puedan convertirse en sus Primeros Voceros.

Que los empleados sean voceros de la organización donde trabajan, debería ser el objetivo central de la Comunicación Corporativa enfocada hacia los Públicos Internos

\section{Obtención de la Información}

Continuando con el proceso de formación de la Imagen Corporativa, las personas acceden a la información de la organización a través de diversos medios. Desde la estrategia comunicacional, se trata de identificar cuáles son los medios y las fuentes de información; y cómo es la relación que los públicos establecen con los diferentes tipos de medios; como también, cuál es el nivel de credibilidad que se adjudica a las diversas fuentes.

Como señala Capriotti (1999), las personas acceden a información de la organización a través de tres vías: información directa, información personal e información mediada.

La información directa corresponde al ámbito de la acción comunicativa de la organización. Y la información personal es precisamente a la que se accede a través de la experiencia personal, cuando se produce un contacto con la organización de modo que no existe la intervención de otro o de grupos.

Este contacto puede ser directo o indirecto. Mientras que en el contacto directo se produce la interacción de personas, en el contacto indirecto la relación se establece por el uso de productos, y/o acceso a servicios.

Usualmente, los individuos establecen contacto con varias organizaciones, siendo algunas de ellas competidoras entre sí; con lo cual además del análisis particular que pueden realizar a partir de cada experiencia, también pueden establecer una comparación de las mismas.

Ante la presencia de productos o servicios similares se establece una etapa de evaluación donde el contacto directo puede constituirse en el factor diferencial que destaque a una organización sobre otra; y en función de lo actuado por el empleado, formar una imagen más sólida y positiva en la mente de los públicos. Pensemos en qué nos pasa, como clientes y/o consumidores, cuando recibimos un trato destacado por parte del empleado de una organización. ¿Y cuándo sucede lo contrario?

La visión actual de la gestión de la Comunicación Interna debe tener en cuenta este rol que cumplen los empleados en la construcción de la Imagen Corporativa; más allá de la función de Calidad de Servicio que se implementan desde las áreas comerciales de las organizaciones. Este rol también puede incluirse como parte de la formación de los empleados como voceros. Por último, se encuentra la información mediada, que es aquella que le llega a la persona a través de otro, individuo u organización, y de los medios masivos de comunicación. Y también, 
se suma como fuente a la red de relaciones y la información que ésta nos brinda a partir de sus propios contactos directos o indirectos (o sea, a su información experimentada).

Estas fuentes que transmiten información, también ejercen influencia. Es por eso que además de identificarlas, debemos conocer el grado de intervención que ejercen en la formación de la Imagen Corporativa.

Como se sabe, los medios de comunicación son fuente de información privilegiada para satisfacer la necesidad de conocimiento de los individuos sobre el entorno; y además, ejercen una importante influencia en la construcción de la realidad de las personas. Por ende, también en la construcción de imagen.

En esta información que llega a través de los medios, encontramos datos acordes a la acción comunicativa de la organización. Lo cual puede generar un refuerzo o un conflicto entre el mensaje directo de la organización y el de los medios.

Cuando se establece el conflicto, debemos recordar el importante efecto que se tienen los medios sobre las personas; efecto que es mayor cuanto menor sea la experiencia directa de la persona con la organización, o menor sea el acceso a información que le llega de manera mediada a través de su red de relaciones.

Entonces, es oportuno señalar ahora a esta otra fuente de información que son la red de relaciones. Las personas interactúan de manera cotidiana y en esa interacción, se produce intercambio de información. Esta información puede influir, de manera intencionada o no, en el receptor dependiendo del nivel de conocimiento y de congruencia ideológica que existe entre el emisor y ese destinatario.

En el siguiente cuadro podemos ver cómo se establecen y relacionan los conceptos que han sido señalados sobre el proceso de formación de la imagen corporativa:

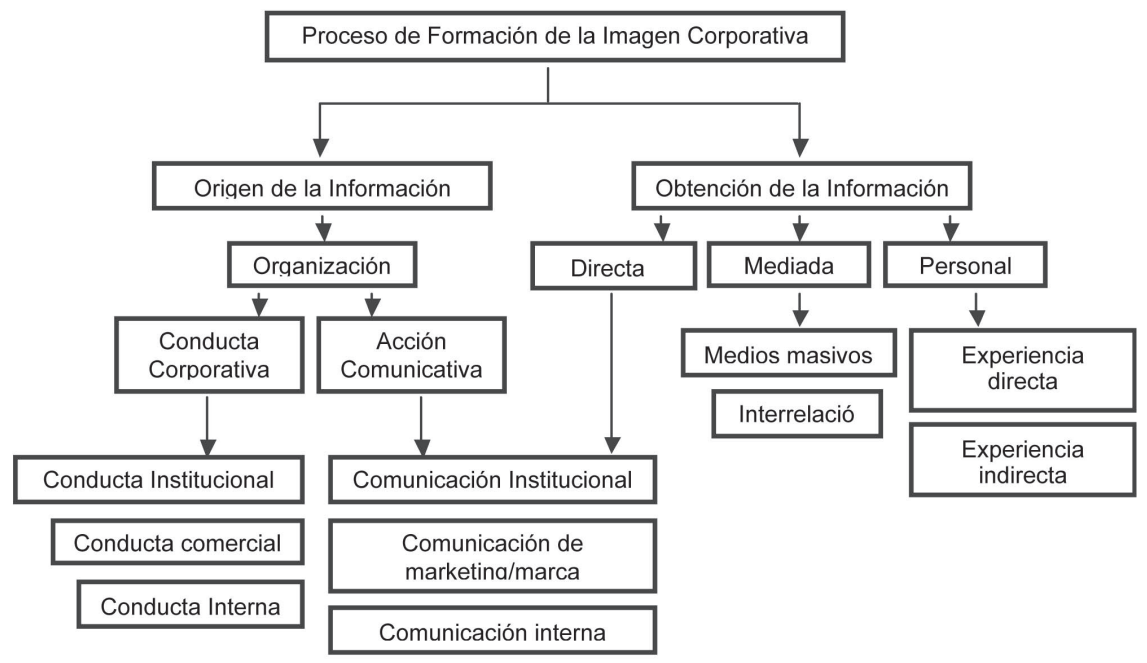

Figura 4: La Comunicación en la Organización.

Fuente: Capriotti, P. (1999). Planificación estratégica de la imagen corporativa. Barcelona: Ariel. 
Se puede encontrar un elemento clave en la importancia del rol de los Públicos Internos en esta construcción cuando jerarquizamos a las diferentes fuentes de información según la credibilidad que se le otorga a las mismas.

Estas fuentes no pueden ser tomadas de manera independiente ya que interactúan en el contexto social en un proceso continuo de relación e interdependencia y se influencian unas a otras. Los individuos suelen encontrarse ante la posibilidad de obtener información a través de las tres fuentes citadas: consumen información que puede ser influida por las redes de relación y experiencias personales. Como a su vez, la información de los medios puede influir sobre la experiencia de los individuos y la información que luego ellos brindan.

Hemos visto cómo los medios son fuente de información privilegiada y además, ejercen una importante influencia en la construcción de la realidad de las personas, especialmente cuando la experiencia directa con la organización es menor o nula; y tampoco se obtiene información a través de la red de relaciones. Pero, ¿qué pasa cuando ocurre lo contrario? ¿Qué sucede cuando las personas establecen un contacto directo o reciben información de otras personas de su confianza que han tenido ese contacto con la organización? ¿Cuál es entonces la importancia e influencia que tiene la información según estas fuentes?

Los individuos toman a estas fuentes de manera complementaria; y otorgan a cada una un grado de credibilidad determinado, tendiendo a aceptar a aquella que posee el mayor grado en comparación con las demás. Y este grado de credibilidad es esencial en la formación de la imagen en la mente de los públicos.

Tal como expresa Capriotti (1999, pp. 109-111), si realizamos una jerarquizacion, la experiencia personal de los individuos sería la fuente de mayor jerarquía. Luego, podemos ubicar a la información que se obtiene de las redes de relaciones, ya que estamos ante otras experiencias personales. Y finalmente, podemos ubicar a la información de los medios.

$\mathrm{Si}$ analizamos porcentualmente, podemos decir que el $66 \%$ de la información que se considera más creíble para la formación de la imagen corporativa es aquella que se encuentra relacionada con el decir y el hacer de los Públicos Internos de la organización. Por un lado, tenemos la experiencia directa del individuo, y por el otro, una experiencia mediatizada dado que la información también refiere a una experiencia personal. Se trata y se parte de situaciones de contacto.

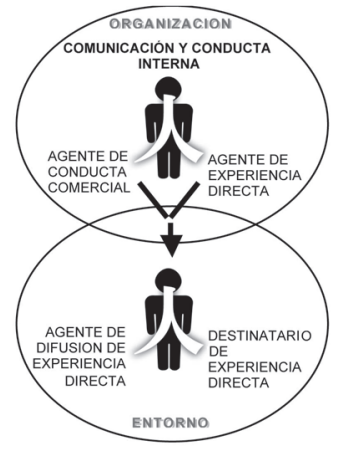

Figura 5: Comunicación, organización y entorno.

Fuente: Elaboración propia. 
Y aún queda una variable por incorporar. Se trata de la información que brinda un individuo a su red de relaciones como miembro de una organización. ¿Qué ocurre cuándo leemos información positiva de una empresa en un medio de comunicación pero recibimos información divergente a través de un individuo que trabaja en esa organización? Se trata de alguien que integra nuestra red de relaciones, el segundo escalón de credibilidad. Sin necesidad de experimentar un contacto directo, consideramos a esta fuente antes que al medio masivo.

¿Están las organizaciones plenamente conscientes de que es cada vez más importante identificar el diferencial de credibilidad que tienen sus propios empleados como fuente de información? Las actuales y nuevas tecnologías permiten que la información de esta categoría de fuente se encuentre cada vez más accesible para el conjunto de relaciones y empiece a pesar en la formación de la Imagen Corporativa de las organizaciones.

\section{Las nuevas tecnologías y el Público Interno}

Hasta ahora vimos cómo la acción comunicativa y la conducta corporativa, las experiencias personales y las interrelaciones, son un ámbito para la influencia de los Públicos Internos en la construcción de la Imagen Corporativa en la mente de los Públicos Externos.

Lo que proponemos ahora es identificar cómo en el ámbito de las nuevas tecnologías, podemos encontrar un escenario peculiar donde se produce un encuentro no mediatizado de estos dos públicos.

Al hablar de nuevas tecnologías debemos comprender que nos encontramos ante la formación de un nuevo paradigma comunicacional, donde a partir del acceso cada vez más universal al ciberespacio, cada individuos puede constituirse en autor, emisor y creador de contenido.

Hoy, cualquier persona desde su computadora puede acceder a diferentes portales de noticias, redes sociales, blogs, wikis; y encontrar allí los medios necesarios para emitir una opinión, comentario, juicio, valoración.

Como dice Joan Costa , existen "toda una serie de medios que permiten a cada uno crear, producir, almacenar, manipular, distribuir y recibir informaciones. Con la tecnología interactiva, todos somos emisores y difusores de mensajes" (2009, p. 25).

En este mundo virtual, se amplía el número de contacto, de relaciones; o sea, el público objetivo de aquel contenido transmitido. Y si antes la opinión tenía incidencia en el entorno cercano, ahora nos encontramos con "mundos de relaciones" de 200, 300 contactos.

Entonces, por un lado, hemos establecido que al jerarquizar las fuentes de información, la experiencia personal de los individuos es la fuente privilegiada y nos encontramos ante un mundo que provee información de manera constante y multiplicada sobre la experiencia de miles de individuos que se vuelcan al mundo virtual.

Y si además, como también hemos establecido anteriormente, podemos ubicar a la información obtenida de las redes de relaciones en el segundo lugar jerarquizado, encontramos en el "boca a boca" virtual una fuente inagotable de información para la construcción de la Imagen Corporativa. Como han expresado Alessandro Barbosa y Federido Rey Lennon en el Cuaderno Nro. 28 de la Facultad de Diseño y Comunicación de la Universidad de Palermo, sobre Relaciones Públicas, esta comunicación "se volvió visible, con el poder suficiente para influir un universo mayor 
de personas y se transformó en una medida importante, tanto cualitativa como cuantitativa, del éxito de un producto, de un servicio o de una campaña". (Lennon y Barbosa, 2009, p. 28) ¿Porqué? Una de las razones es porque actualmente cualquier consumidor y/o cliente tiene la posibilidad de expresar su opinión en el ciberespacio acerca de su experiencia con el producto consumido o el servicio contratado. Quienes están habituados a estos espacios de la Web 2.0 saben que se puede encontrar información sobre un determinado producto o servicio; que alguien ha decidido compartir; generalmente para advertir sobre algún mal servicio, problemas de un producto o incumplimiento de la organización. Y también se pueden encontrar espacios donde se plantean preguntas y búsquedas de información de manera proactiva como antesala a la adquisición del producto o contratación del servicio. Aquella información que antes se recababa en un entorno físico acotado, hoy se plantea en el universo virtual de múltiples contactos. La información llega no mediatizada por la organización ni por los medios de comunicación; y generalmente con referencia a experiencias personales. Y esta información, se hace accesible a un mundo incalculable de personas de manera duradera...

En este contexto, las organizaciones aún no han establecidos modelos de gestión para establecer un rol adecuado en este nuevo paradigma; por lo que esta información y este comportamiento todavía aparece más como una amenaza que como una oportunidad.

Como vimos, esta emisión de contenido a partir de experiencias personales es la información más valorada respecto a la información de la organización. Y, ¿qué grado de credibilidad y valoración adquiere cuando esa misma información en lugar de ser emitida por un cliente o consumidor es emitida por un empleado? ¿Están las organizaciones pensando en este potencial rol del Público Interno?

Volvemos sobre la idea inicial: ahora más que nunca, que los empleados sean voceros de la organización donde trabajan, debe ser el objetivo central de la Comunicación Corporativa enfocada hacia los Públicos Internos.

Y es una visión integradora y a largo plazo como la del DirCom, aquella que puede percibir este potencial para incluirlo adecuadamente en la acción estrategia que le corresponde a la Comunicación Corporativa.

\section{Referencias Bibliográficas}

Barbosa Lima, A. y Rey Lennon, F. (2009). La Web 2.0: el nuevo espacio público. En Cuaderno 28. Relaciones Públicas 2009. Radiografía: proyecciones y desafios. Cuadernos del Centro de Estudios en Diseño y Comunicación (ensayos). Buenos Aires: Facultad de Diseño y Comunicación-Universidad de Palermo.

Bernays, E. (1958). Public Relations Idea Book. USA: Printers'Ink Pub. Co.

Capriotti, P. (2009). Branding Corporativo. Santiago de Chile: Colección Libros de la Empresa. (1999). Planificación estratégica de la imagen corporativa. Barcelona: Ariel.

Costa, J. (2009). El DirCom hoy. Dirección y Gestión de la Comunicación en la nueva economía. Barcelona: Costa PuntoCom Editor.

Rey Lennon, F. (1999) Edward Bernays. El hombre que inventó las relaciones públicas. Buenos Aires: EDUCA. 
Summary: This article reflects about the internal public with the aim to define its role and relevance in the organizations; as as much contributor of the process of origin and obtaining of information, like also in the construction of the mental mental structures of the external public that it precedes to the generation of the organizational bonds. For this, internal communication, internal public, organizational culture and corporative communication, have to be tied to image construction process. Finally, it deepens on the new technologies and the new communicational paradigm, in which the internal public definitively assumes its role as a spoke person.

Key words: communication people - Corporate communications - corporate image - Dircom external public - institutional communication - internal public - new technologies - organizational communication - Public Relations - spokeperson.

Resumo: Este artigo reflexiona a respeito dos públicos da organização como destinatários da gestão das relações públicas, e em particular do público interno, com o objetivo de assinalar seu papel e a importância nas organizações; tanto como participantes do processo de origem e obtenção de informação, como também na construção das estruturas mentais cognitivas dos públicos externos que antecede à geração dos vínculos organizacionais. Para isto, necessariamente se vincula comunicação interna, públicos internos, cultura organizacional e comunicação corporativa, e como estes impactam na construção de uma imagem positiva. Finalmente, aprofunda sobre as novas tecnologias e em mudança de paradigma comunicacional, no que o público interno definitivamente assume o papel de porta-voz.

Palavras chave: Comunicação institucional - comunicação organizacional - comunicadores Comunicações corporativas - Dircom - imagem corporativa - novas tecnologías - porta-voz público externo - público interno - Relações Públicas.

${ }^{*}$ ) Licenciada en Relaciones Públicas de la Universidad Nacional de Lomas de Zamora y egresada del Programa de Comunicación Institucional de la Universidad Austral. Especialista en Comunicación Organizacional. [Ver CV completo en la página 141] 